\title{
V240, NUEVA VARIEDAD SUBTROPICAL DE MAÍZ COMITECO
}

\section{V240, A NEW SUBTROPICAL COMITECO MAIZE VARIETY}

\author{
Bulmaro Coutiño Estrada ${ }^{1}$ * y Víctor A. Vidal Martínez ${ }^{2}$
}

\begin{abstract}
'Instituto Nacional de Investigaciones Forestales, Agrícolas y Pecuarias (INIFAP), Campo Experimental Centro de Chiapas, Ocozocoautla, Chiapas, México. ${ }^{2}$ INIFAP, Campo Experimental Santiago Ixcuintla, Santiago Ixcuintla, Nayarit, México.

*Autor de correspondencia (coutino.bulmaro@inifap.gob.mx)
\end{abstract}

El cultivo de maíz (Zea mays L.) en la Meseta Comiteca de Chiapas es muy importante, pues en el año 2018 se cultivaron 95,111 ha de maíz, con una producción de 180,733 t (SIAP, 2020), casi en su totalidad con variedades criollas de la raza Comiteco y con rendimientos promedio de 1.9 t ha $^{-1}$. En el año 1994 el Instituto Nacional de Investigaciones Forestales, Agrícolas y Pecuarias (INIFAP) liberó la variedad de maíz denominada V-229 proveniente del primer ciclo de selección combinada realizado en la población Comiteca Blanca (Coutiño et al., 2004). Con semilla de esta variedad, en el año 1998 se estableció un lote aislado, donde fueron seleccionadas 155 familias de medios hermanos, a partir de las cuales se hicieron seis ciclos de selección, mediante el uso del método modificado mazorca por surco de medios hermanos (Compton y Comstock, 1976; Hallauer y Miranda, 1981; Lonnquist, 1964), también conocido como selección combinada (Márquez, 1985).

La evaluación de las 155 familias de medios hermanos, más la población $\vee-229$, se realizó en dos o tres localidades bajo el diseño experimental látice simple rectangular $13 \times 12$, en parcelas de un surco con 22 plantas. La recombinación genética de las familias superiores, para la obtención de nuevas 155 familias y la formación de las variedades experimentales con las 10 mejores familias por localidad y por región, se hicieron al año siguiente, por lo que un ciclo de selección se obtuvo en dos años (12 años en total). Durante este proceso, las variedades experimentales obtenidas en cada ciclo fueron evaluadas para conocer el avance o ganancia genética de los ciclos respectivos de selección. Como resultado de estas pruebas, se formó un compuesto genético con las variedades experimentales Regional C6, Comitán C6 y Teopisca C6, que superaron en rendimiento a las variedades de los ciclos de selección anteriores, y se le denominó Comiteca Blanca C6.
Esta nueva variedad se evaluó durante los ciclos agrícolas de los años 2015, 2016 y 2017 con productores cooperantes de La Meseta Comiteca, Chiapas, donde se obtuvieron rendimientos promedio de $4.8 \mathrm{t} \mathrm{ha}^{-1}$, que al compararse con las variedades nativas testigo, la Comiteca Blanca C6 produjo 9.6 \% más de grano, tuvo menos acame, similar cobertura de mazorca, que impide pudriciones de grano, y similar calidad de grano. Bajo buenas condiciones de lluvia, como las del año 2017, y en suelos de buena calidad del municipio de La Trinitaria, esta variedad produjo $7.8 \mathrm{t} \mathrm{ha}^{-1}$.

En febrero de 2019 se le registró en el Catálogo Nacional de Variedades Vegetales del Servicio Nacional de Inspección y Certificación de Semillas (SNICS) de la SADER con el nombre de V240 y número provisional 3862-MAZ2070-120219/C, en octubre del mismo año se obtuvo el Título de Obtentor con número de registro 2316.

Las plantas de $\mathrm{V} 240$ crecen de 2.50 a 3.08 m y la mazorca se inserta de 1.45 a 1.89 m; inician la liberación de polen de los 95 a 100 d, llegan a etapa de elote a los 136 d, el cual es de $20 \mathrm{~cm}$ de largo, peso de 205 g, contenido de sólidos solubles de $9{ }^{\circ}$ Brix y alcanzan su madures fisiológica a los $200 \mathrm{~d}$.

Las mazorcas son de forma cónica-cilíndrica, de 18 a $25 \mathrm{~cm}$ de largo y de 4 a $5 \mathrm{~cm}$ de diámetro, tienen 10 hileras de grano, aunque se encuentran también de 12 y 14 , y cada hilera puede tener de 30 a 45 granos, los cuales son de endospermo color blanco cremoso, de textura semidentada principalmente, pero se encuentran también granos semicristalinos (Figura 1); los granos contienen $11.6 \%$ de proteína, lo que los hacen más nutritivos en comparación con otras variedades; su peso hectolítrico es de $70 \mathrm{~kg} \mathrm{hL}^{-1}$. Puede cultivarse en los Distritos de Desarrollo Rural de San Cristóbal de Las Casas y de Comitán, en 


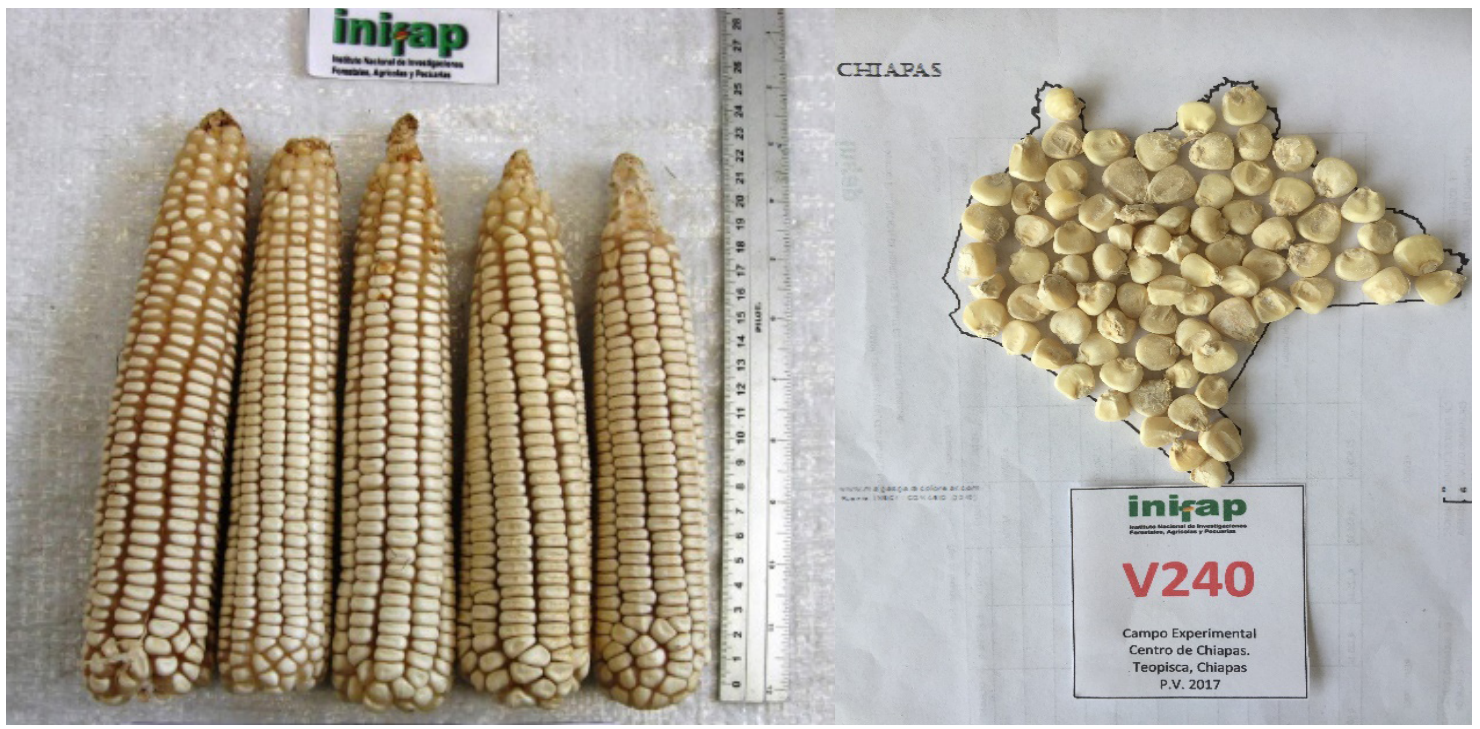

Figura 1. Aspecto de mazorca y grano de la variedad de maíz V240

los municipios de Amatenango del Valle, Teopisca, Las Rosas, Huixtán, Comitán, Las Margaritas, La Trinitaria, La Independencia y Tzimol, que tienen clima del tipo $A(C)$ w, semicálido subhúmedo con lluvias en verano, y en otras regiones similares con alturas de 1200 a 1800 msnm y precipitaciones mayores de $800 \mathrm{~mm}$, tanto en siembras del sistema pul-jhá (echar agua), como de temporal.

El INIFAP tiene semilla original disponible para producir las categorías básica y registrada que requieran las empresas semilleras para la producción y comercialización de semilla categoría certificada, conforme a las recomendaciones de aislamiento por espacio o tiempo del SNICS y haciendo los desmezcles adecuados (Coutiño, 1993).

\section{AGRADECIMIENTOS}

Los autores agradecen a la Fundación Produce Chiapas, A. C. el financiamiento otorgado al Proyecto 2685: "Mejoramiento poblacional de las razas de maíz Comiteco y Tuxpeño para formar variedades mejoradas" el cual apoyó parcialmente estos trabajos durante los años 2001 a 2004.

\section{BIBLIOGRAFÍA}

Compton W. A. and R. E. Comstock (1976) More on modified ear-to-row selection in corn. Crop Science 16:122, https://doi.org/10.2135/ cropsci1976.0011183X001600010034x

Coutiño E. B. (1993) Normas y técnicas para producir semilla certificada de variedades de maíz. Folleto Técnico No. 7. Campo Experimental Centro de Chiapas. Instituto Nacional de Investigaciones Forestales, Agrícolas y Pecuarias. Ocozocoautla, Chiapas, México. 33 p.

Coutiño E. B., E. Betanzos M., A. Ramírez F. y N. Espinosa P. (2004) V-229 y $\vee-231 \mathrm{~A}$, primeras variedades mejoradas de maíz de la raza Comiteco. Revista Fitotecnia Mexicana 27:295-296.

Hallauer A. R. and J. B. Miranda (1981) Quantitative Genetics in Maize Breeding. The lowa State University Press. Ames, lowa. 468 p.

Lonnquist J. H. (1964) A modification of the ear-to-row procedure for the improvement of maize populations. Crop Science 4:227228, https://doi.org/10.2135/cropsci1964.0011183X0004000 $20033 x$

Márquez S. F. (1985) Genotecnia Vegetal. Tomo I. Métodos, Teoría, Resultados. AGT Editor. México, D. F. 356 p.

SIAP, Servicio de Información Agroalimentaria y Pesquera (2020) Anuario estadístico de la producción de maíz en el estado de Chiapas. Ciclo agrícola 2018. Secretaría de Agricultura y Desarrollo Rural Ciudad de México. https://nube.siap.gob.mx/cierreagricola/ (Mayo 2020). 